\title{
WOS-ELM-Based Double Redundancy Fault Diagnosis and Reconstruction for Aeroengine Sensor
}

\author{
Zhen Zhao, ${ }^{1}$ Zhexu Liu, ${ }^{1}$ Yigang Sun, ${ }^{2}$ and Jingya Liu $^{3}$ \\ ${ }^{1}$ College of Electronic Information and Automation, Civil Aviation University of China, Tianjin 300300, China \\ ${ }^{2}$ College of Aerospace Engineering, Civil Aviation University of China, Tianjin 300300, China \\ ${ }^{3}$ Tianjin Binhai International Airport, Tianjin 300300, China
}

Correspondence should be addressed to Yigang Sun; caucsyg@163.com

Received 13 June 2017; Revised 8 September 2017; Accepted 13 September 2017; Published 26 November 2017

Academic Editor: Zhixing Cao

Copyright (c) 2017 Zhen Zhao et al. This is an open access article distributed under the Creative Commons Attribution License, which permits unrestricted use, distribution, and reproduction in any medium, provided the original work is properly cited.

\begin{abstract}
In order to diagnose sensor fault of aeroengine more quickly and accurately, a double redundancy diagnosis approach based on Weighted Online Sequential Extreme Learning Machine (WOS-ELM) is proposed in this paper. WOS-ELM, which assigns different weights to old and new data, implements weighted dealing with the input data to get more precise training models. The proposed approach contains two series of diagnosis models, that is, spatial model and time model. The application of double redundancy based on spatial and time redundancy can in real time detect the hard fault and soft fault much earlier. The trouble-free or reconstructed time redundancy model can be utilized to update the training model and make it be consistent with the practical operation mode of the aeroengine. Simulation results illustrate the effectiveness and feasibility of the proposed method.
\end{abstract}

\section{Introduction}

To serve as the heart of an aircraft, it is vital to ensure the security of the aeroengine $[1,2]$. One way to determine whether the aeroengine is running with a fault or not is based on the data collected from the embedded sensors, which must be correct and supply the actual information. Regarding this, it is very important to employ a precise diagnosis of the aeroengine sensors' faults.

Many algorithms have been presented to diagnose the faults of the aeroengine sensors, such as Kalman filter [3], Support Vector Machine [4, 5], Neural Network [6], and wavelet [7]. Kalman filter is a widely used fault detection method. By applying Kalman filter to filter aeroengine sensors' outputs, a set of residual sequences is therefore obtained and analyzed to discover the faulty sensor. In [3], a bank of Kalman filters are used to detect and isolate sensors' faults, and another robust Kalman filter is used to detect any statistics changes happening to the sensors or the actuators. The premise of this method is to build an accurate aeroengine state variable model. However, sometimes, it is difficult or impossible to establish an accurate mathematical model.
Least squares support vector machine (LS_SVM) online prediction is proposed in [4]. Through residual, which is obtained by comparing the actual outputs of the sensor and those of LS_SVM prediction model established using the realtime sensors' outputs, the sensors' faults can be detected in real-time. Wireless sensor fault diagnosis based on RBF neural network and ant colony optimization is presented in [6]. Since the connection weights, the hidden centers, and the widths have an important influence on the classification performance of the RBF neural network in the learning process, parameter selection has a close relation with the diagnosis precision. The common disadvantage of these algorithms is that they all need to set up a great deal of parameters, which increase certain difficulty for their application.

Extreme Learning Machine (ELM) [8], proposed by Huang et al., can solve this problem well. ELM only needs to set up two parameters, that is, number of hidden layer neurons and activation function [8], which may provide a more convenient way to build diagnosis model for aeroengine sensors. Furthermore, it shows faster training speed and higher model's accuracy than BP Neural Network $[9,10]$. Nevertheless, ELM algorithm requires training all input data all 


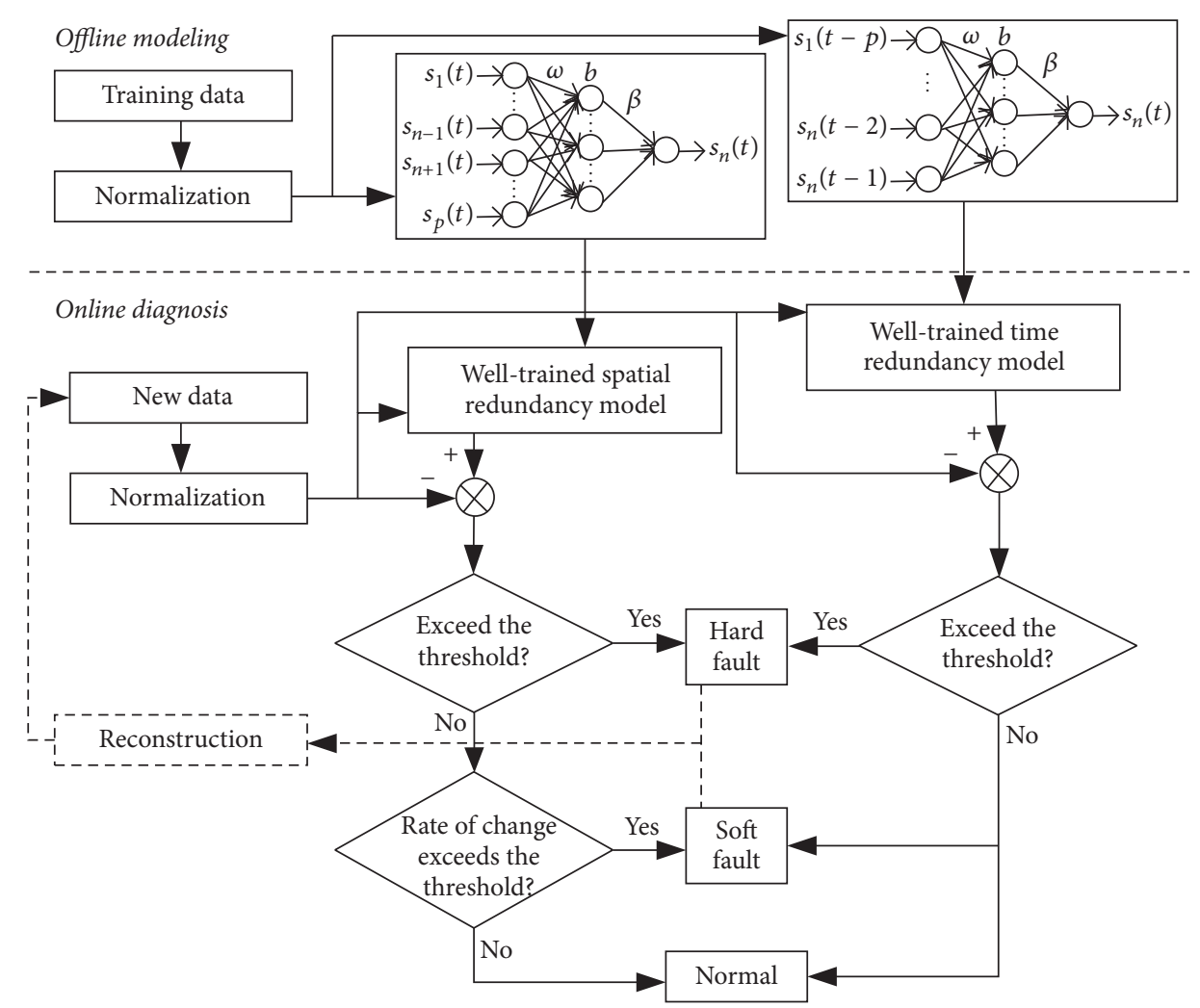

FIGURE 1: WOS-ELM-based double redundancy FDR method.
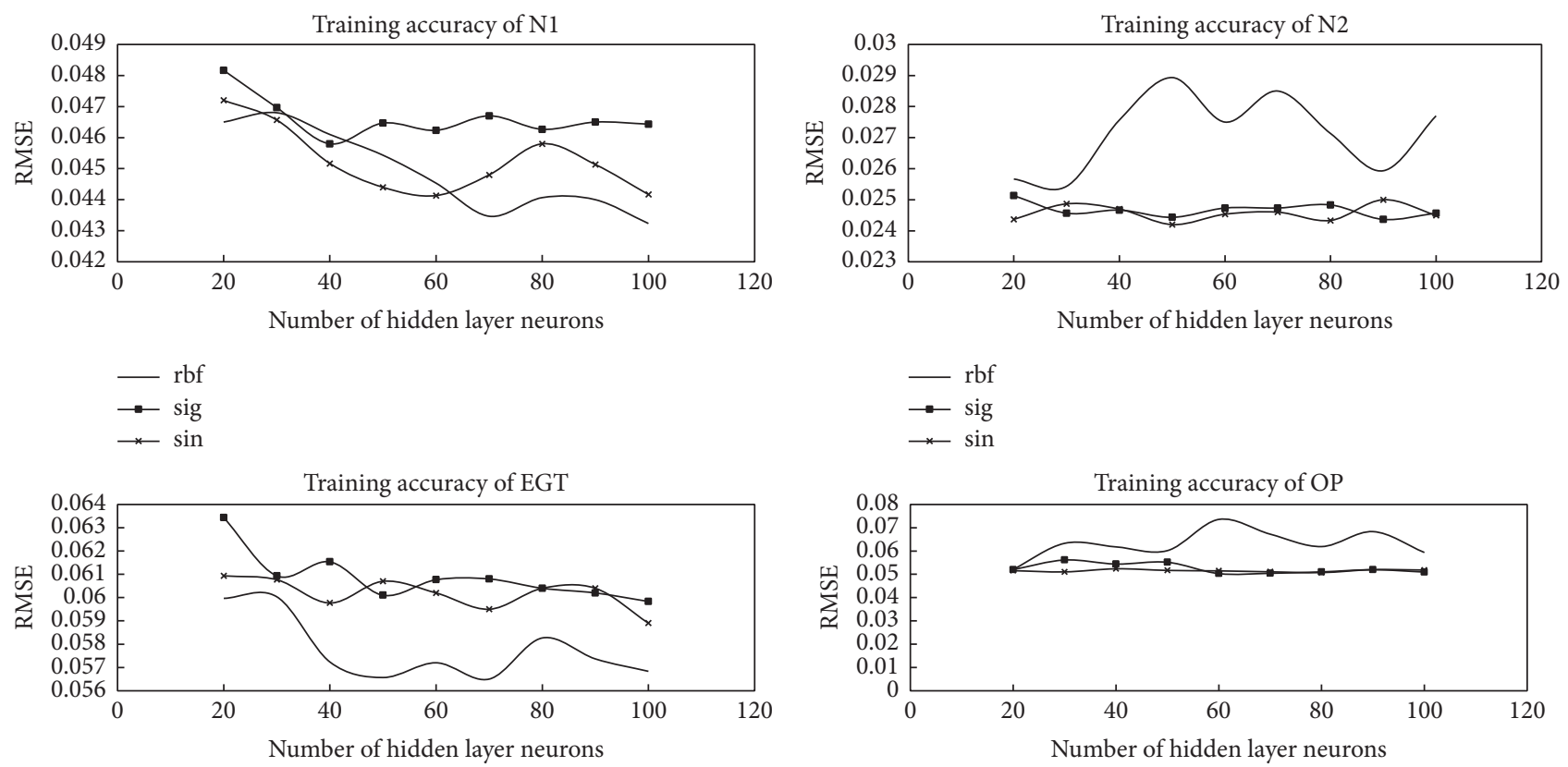

$-\mathrm{rbf}$
$\rightarrow-\operatorname{sig}$
$\rightarrow \sin$

$\longrightarrow$ rbf
$\rightarrow-\operatorname{sig}$
$-\quad \sin$

FIgURE 2: Training accuracy with different activation functions. 

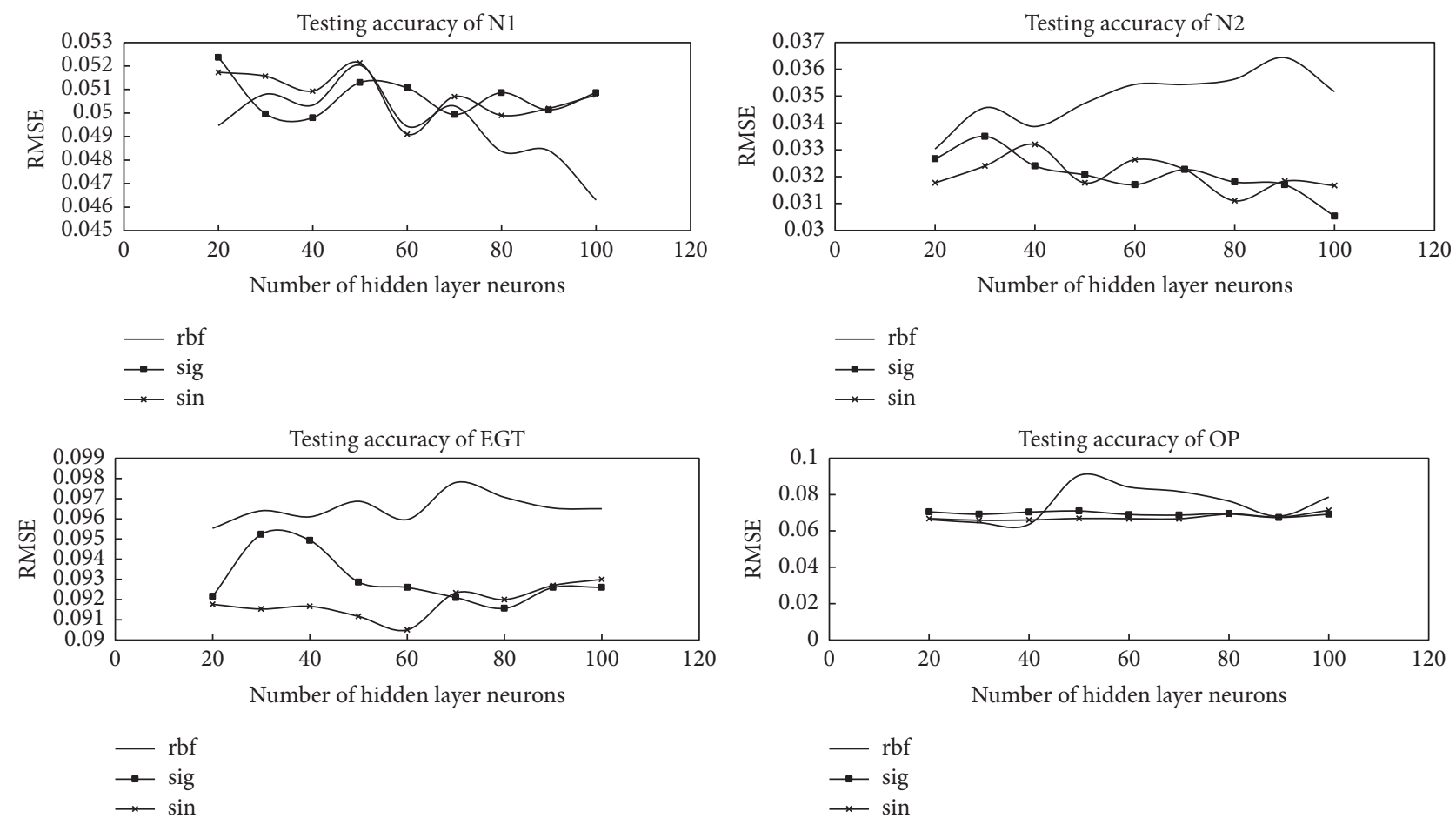

FIGURE 3: Testing accuracy with different activation functions.

at once. Sometimes, we cannot obtain all the sampling data at one time. When the new data comes, it has to train the model using the whole data again, which may increase its training time. Online Sequential Extreme Learning Machine (OS-ELM) [11] can enable the training data to be trained one-by-one or chunk-by-chunk, which is a better choice for real-time fault diagnosis. In recent years, OS-ELM algorithm has been used in the field of fault diagnosis widely. Yin et al. used OS-ELM algorithm to realize fault diagnosis of rotating machinery [12]. Multiple model classifiers were established, and simulation results illustrated its good ability in classification. A fault diagnosis method based on weighted dissolved extreme learning machine (WELM) is presented for transformer in [13]. WELM algorithm is proposed to deal with data imbalance in dissolved gas analysis data. As an improved algorithm, ImOs-ELM is presented in [14] to realize aeroengine sensor fault diagnosis, which has great advantages in real-time capability and accuracy. However, the aeroengine sensor model is known in advance. Considering that we only have the sensors' output data when the plane flights are under normal condition, and lack of the data when sensors' faults are happening, the achievement of aeroengine sensor fault diagnosis based on OS-ELM algorithm shows a good ability of generalization. In terms of aeroengine sensor data, sensor output data obtained in different flight phases keeps changing greatly. Therefore, weighted dealing with the old and new data can increase accuracy of diagnosis model. For this reason, this paper utilizes WOS-ELM $[15,16]$ to build the diagnosis model.
Generally speaking, the fault occurring in sensor can be categorized into two typical forms. One type is "hard fault," which shows obvious fault symptom and may cause substantial losses, severe safety, significant casualties, and so on. Due to its obvious fault symptom, it is relatively easy to be detected and diagnosed. The other is "soft fault," also named incipient fault, whose fault symptom is very small and the system has not been affected at the beginning, and the fault symptom gradually becomes obvious as time goes on, ultimately causing meltdowns. For "soft fault," if it could be detected and diagnosed as early as possible, subsequent safety risk, system damage, and casualties would be avoided. However, it is difficult to identify these weak fault symptoms from the noises to detect and diagnose "soft fault" at the beginning. Therefore, it is more challenging to detect and diagnose "soft fault" in practical applications.

Considering the nonlinear characteristics of aeroengine sensors' relation and the advantage of ELM, this paper introduces OS-ELM to detect and diagnose sensor faults. To ensure the modeling accuracy, WOS-ELM algorithm, assigning different weights to the old and new data, is introduced to update the training model and promotes training model closer to the real status of the aeroengine. To improve the detection and diagnosis precision and timeliness and realize multisensor composite faults, a double redundancy strategy, that is, spatial redundancy and time redundancy, is utilized. Combining ELM and double redundancy strategy, a WOS-ELM-based double redundancy fault diagnosis and reconstruction approach is presented to detect and diagnose 

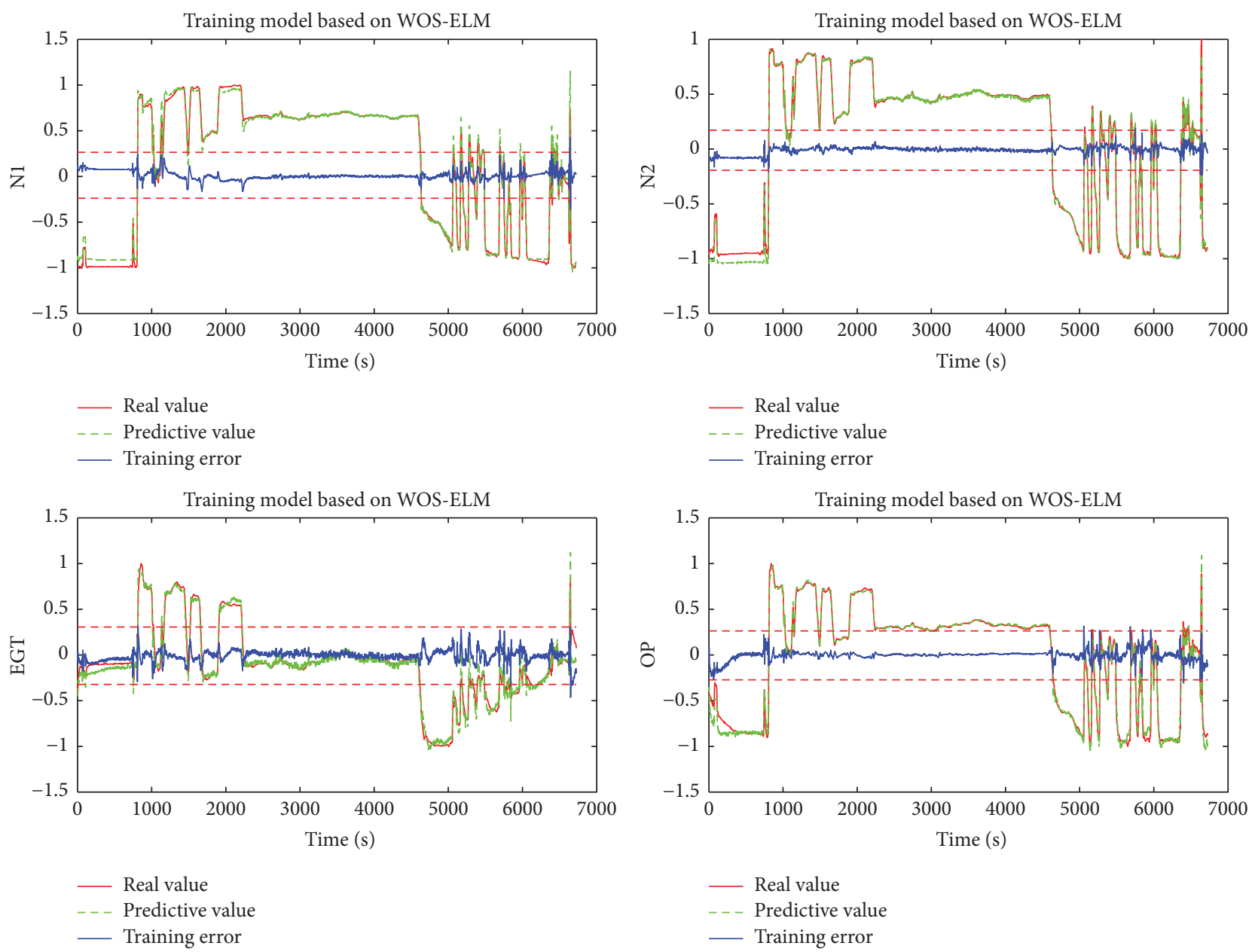

FIGURE 4: Training results for sensors under normal state.

both "hard fault" and "soft fault" for the aeroengine sensor. The main contributions of this paper include the following:

(1) A WOS-ELM-based double redundancy fault diagnosis and reconstruction approach is proposed for aeroengine sensor.

(2) Intermediate parameters are deduced for the WOSELM algorithm.

(3) Through the proposed approach, not only single sensor fault, but also multiple sensor faults can be detected and diagnosed.

The rest of this paper is organized as follows. Section 2 describes Weighted Online Sequential Extreme Learning Machine (WOS-ELM). Section 3 depicts the WOS-ELMbased double redundancy fault diagnosis and reconstruction (FDR) method for aeroengine sensor. Section 4 discusses the simulation result of aeroengine sensor fault diagnosis and reconstruction. Section 5 draws conclusions for the whole paper.

\section{WOS-ELM}

Considering that the training data may arrive chunk-bychunk or one-by-one (a special case of chunk), Online
Sequential Extreme Learning Machine (OS-ELM) was proposed by Liang et al. [11] for single-hidden layer feedforward neural network (SLFN) with additives and RBF hidden nodes. Given a series of arbitrary independent samples $\left(x_{i}, y_{i}\right) \in$ $R^{n} \times R^{m}, i=1,2, \ldots, N$, the actual outputs of the SLFN with $L$ hidden nodes for these $N$ samples are given as follows [11].

$$
f_{L}\left(x_{j}\right)=\sum_{i=1}^{L} \beta_{i} G\left(\omega_{i}, b_{i}, x_{j}\right)=y_{j}, \quad j=1,2, \ldots, N
$$

where $\omega_{i}$ is the input weight vector connecting the input layer neuron to the $i$ th hidden neuron, $b_{i}$ is the bias of the $i$ th hidden node, $\beta_{i}$ is the output weight vector connecting the $i$ th hidden neuron to the output layer neuron, $G\left(\omega_{i}, b_{i}, x_{j}\right)$ is the output of the $i$ th hidden neuron with respect to the input $x_{j}$, and $L$ is the number of hidden nodes.

OS-ELM neglects the time factor when dealing with the training data. It means that old and new data are thought to be the same in reflecting the operating statuses of some equipment (i.e., aeroengine sensors in this paper). WOSELM, considering the time factor and endowing different weights to data sampled at different time period, is an improvement of OS-ELM. The same as OS-ELM algorithm, 

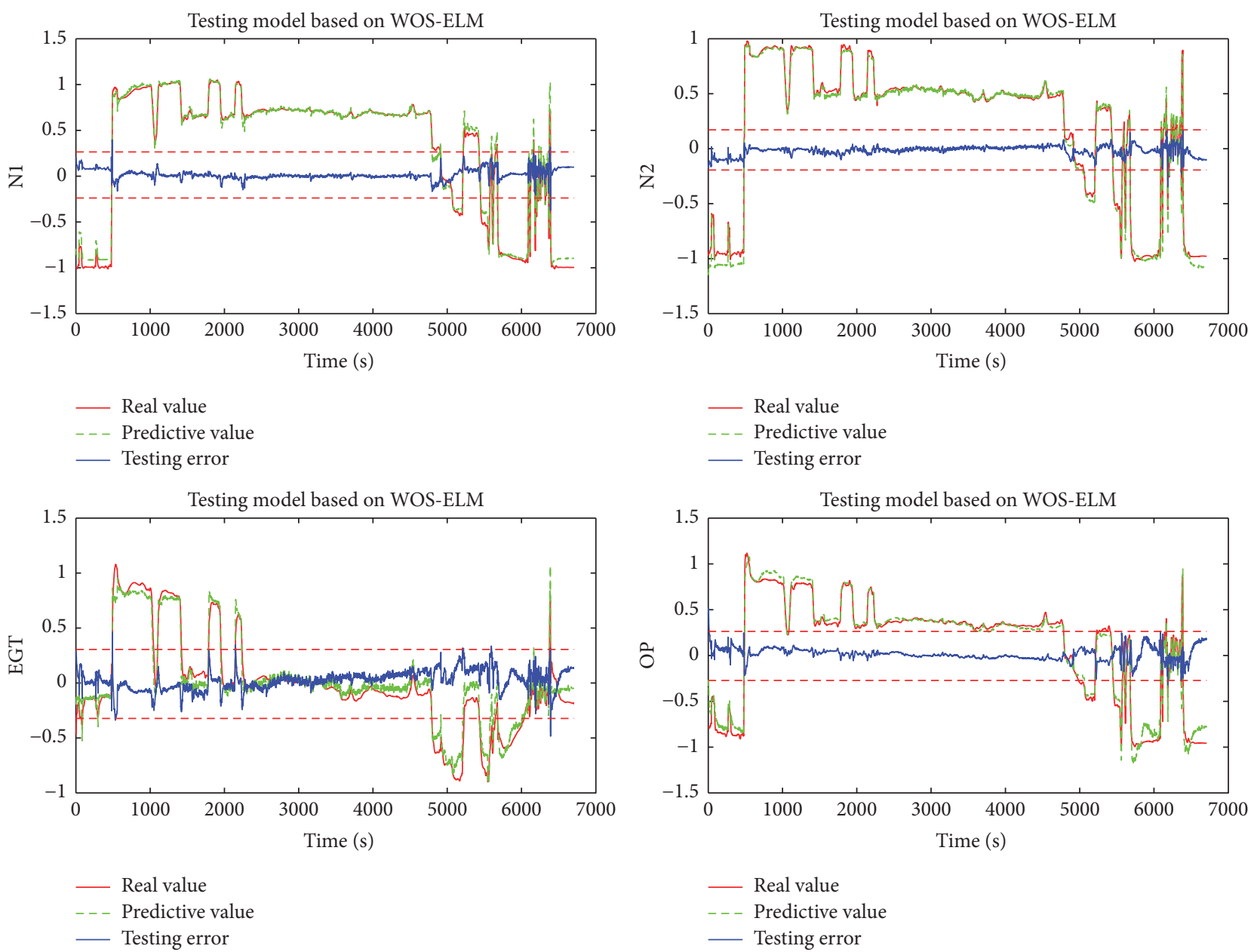

FIGURE 5: Testing results for sensors under normal state.

WOS-ELM can also be divided into two phases, that is, initialization phase and sequential learning phase.

2.1. Initialization Phase. Using a small chunk of training data $\aleph_{0}=\left\{\left(x_{i}, y_{i}\right)\right\}_{i=1}^{N_{0}}$ from the given training set $\aleph=$ $\left\{\left(x_{i}, y_{i}\right) \mid x_{i} \in R^{n}, y_{i} \in R^{m}, i=1, \ldots, N\right\}$ for the initial learning, $N_{0}$ respects the number of initial data, and $N_{0} \geq L$. The initialization phase of WOS-ELM includes four steps as follows.

Step 1. Randomly assign input weight $\omega_{i}$ and bias of hidden neuron $b_{i}(i=1, \ldots, L)$ and give activation function and number of hidden nodes $L$. Normally, different number of hidden nodes and activation function show different precision, which will be proved in the following simulation.

Step 2. Calculate the initial hidden layer output matrix $H_{0}$.

$$
H_{0}=\left[\begin{array}{ccc}
G\left(\omega_{1}, b_{1}, x_{1}\right) & \cdots & G\left(\omega_{L}, b_{L}, x_{1}\right) \\
\vdots & \ddots & \vdots \\
G\left(\omega_{1}, b_{1}, x_{N_{0}}\right) & \cdots & G\left(\omega_{L}, b_{L}, x_{N_{0}}\right)
\end{array}\right]_{N_{0} \times L} .
$$

Step 3. Deduce the initial output weight $\beta^{(0)}$.

$$
\beta^{(0)}=P_{0} H_{0}^{T} Y_{0}
$$

where $P_{0}=\left(H_{0}^{T} H_{0}\right)^{-1}$, and $Y_{0}=\left[y_{1} \cdots y_{N_{0}}\right]_{N_{0} \times m}^{T}$.

Step 4. Set $K_{k}=H_{k}^{T} H_{k}=P_{k}^{-1}(k=0)$.

2.2. Sequential Learning Phase. For the $(k+1)$ th chunk of new data, $\aleph_{k+1}=\left\{\left(x_{i}, y_{i}\right)\right\}_{i=\left(\sum_{j=0}^{k} N_{j}\right)+1}^{\sum_{j=0}^{k+1} N_{j}}\left(N_{k+1}\right.$ stands for the number of data in the $(k+1)$ th chunk); the sequential learning phase is as in the following steps.

Step 1. Calculate the $(k+1)$ th partial hidden layer output matrix $H_{k+1}$.

$$
\begin{aligned}
& H_{k+1} \\
& =\left[\begin{array}{ccc}
G\left(\omega_{1}, b_{1}, x_{\left(\sum_{j=0}^{k+1} N_{j}\right)+1}\right) & \cdots & G\left(\omega_{L}, b_{L}, x_{\left(\sum_{j=0}^{k+1} N_{j}\right)+1}\right) \\
\vdots & \ddots & \vdots \\
G\left(\omega_{1}, b_{1}, x_{\sum_{j=0}^{k+1} N_{j}}\right) & \cdots & G\left(\omega_{L}, b_{L}, x_{\sum_{j=0}^{k+1} N_{j}}\right)
\end{array}\right]_{N_{k+1} \times L}
\end{aligned}
$$



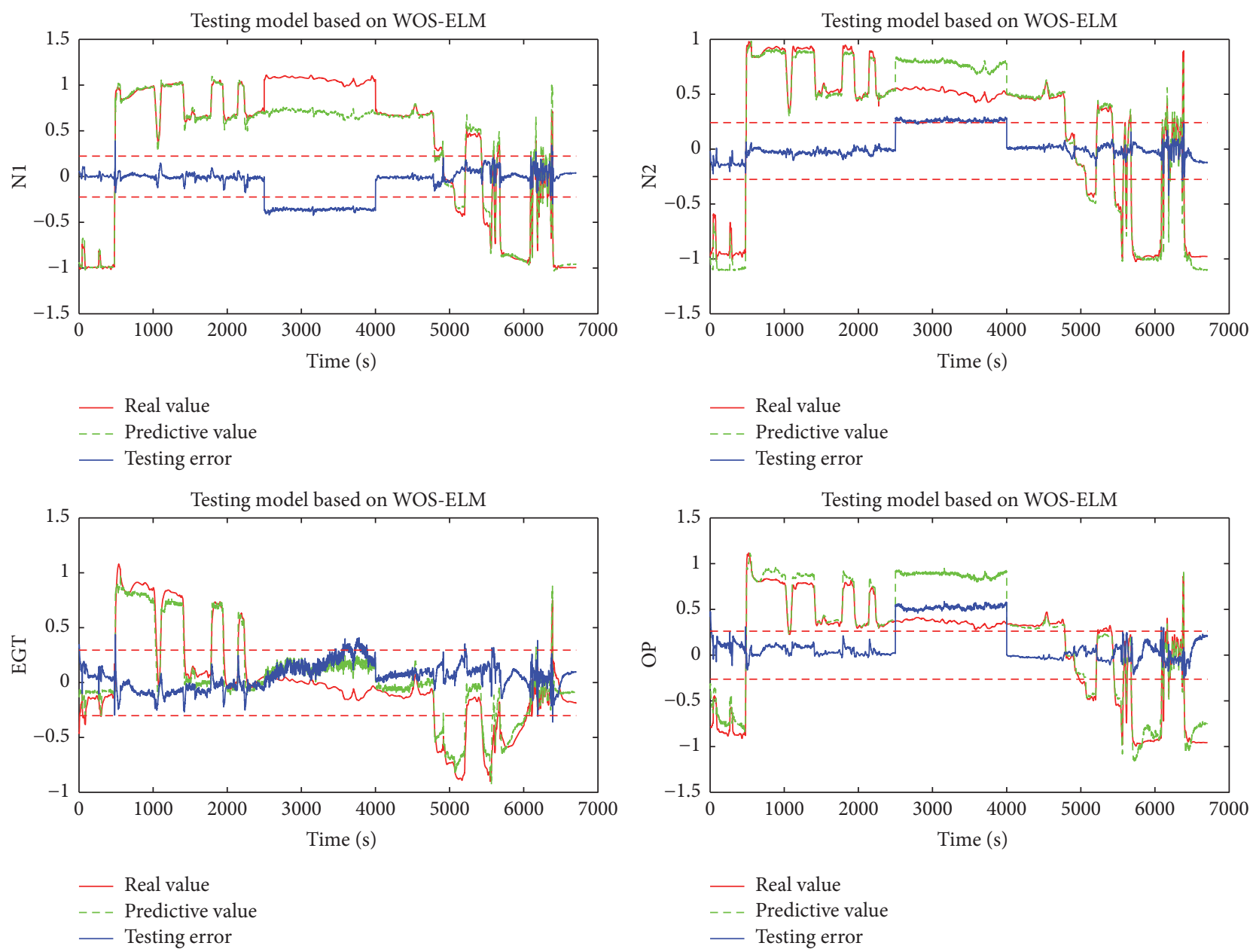

Figure 6: Results of spatial redundancy submodels for step fault of N1.

Step 2. Calculate the $(k+1)$ th output weight $\beta^{(k+1)}$.

The target output can be expressed as

$$
Y_{k+1}=\left[y_{\left(\sum_{j=0}^{k} N_{j}\right)+1} \cdots y_{\sum_{j=0}^{k+1} N_{j}}\right]_{N_{k+1} \times m}^{T} .
$$

In terms of OS-ELM algorithm, $K_{k+1}$ can be expressed as follows.

$$
K_{k+1}=\left[\begin{array}{c}
H_{k} \\
H_{k+1}
\end{array}\right]^{T}\left[\begin{array}{c}
H_{k} \\
H_{k+1}
\end{array}\right]=K_{k}+H_{k+1}^{T} H_{k+1} .
$$

From (6), it can be seen that OS-ELM algorithm handles new data and old data with equal weight, which ignores the influence of time factor. Considering the fact that sensors' output data often changes continuously and greatly when the plane is in the different flight phases, this paper utilizes weighted method to deal with the input data to improve the accuracy of the diagnosis model. Thus, $K_{k+1}$ in the WOSELM can be described as follows.

$$
K_{k+1}=\left[\begin{array}{l}
\lambda H_{k} \\
H_{k+1}
\end{array}\right]^{T}\left[\begin{array}{l}
\lambda H_{k} \\
H_{k+1}
\end{array}\right]=\lambda^{2} K_{k}+H_{k+1}^{T} H_{k+1},
$$

where $\lambda$ stands for weight of the old data, whose value can be determined according to the root mean square error (RMSE) at previous step. RMSE can be written as

$$
\mathrm{RMSE}=\sqrt{\frac{1}{n} \cdot \sum_{i=1}^{n} d_{i}^{2}}
$$

where $d_{i}$ is the deviation between the predicted values of the model and the real values. Define RMSE $\in(a, b)(a, b$ can be set according to practical application). When $\operatorname{RMSE}<a, \lambda=$ 1.005; when RMSE $>b, \lambda=0.995$; else $\lambda=1$. Then, $K_{k+1}^{-1}$ can be deduced as

$$
\begin{aligned}
& K_{k+1}^{-1}=\left(\lambda^{2} K_{k}+H_{k+1}^{T} H_{k+1}\right)^{-1}=\frac{1}{\lambda^{2}}\left(K_{k}^{-1}\right. \\
& \left.\quad-K_{k}^{-1} H_{k+1}^{T}\left(\lambda^{2} I+H_{k+1} K_{k}^{-1} H_{k+1}^{T}\right)^{-1} H_{k+1} K_{k}^{-1}\right) .
\end{aligned}
$$
derive

Considering that $K_{k+1}^{-1}=P_{k+1}$ and $K_{k}^{-1}=P_{k}$, we can

$$
P_{k+1}=\frac{1}{\lambda^{2}}\left(P_{k}-\frac{Q_{k} Q_{k}^{T}}{\lambda^{2} I+H_{k+1} Q_{k}}\right),
$$



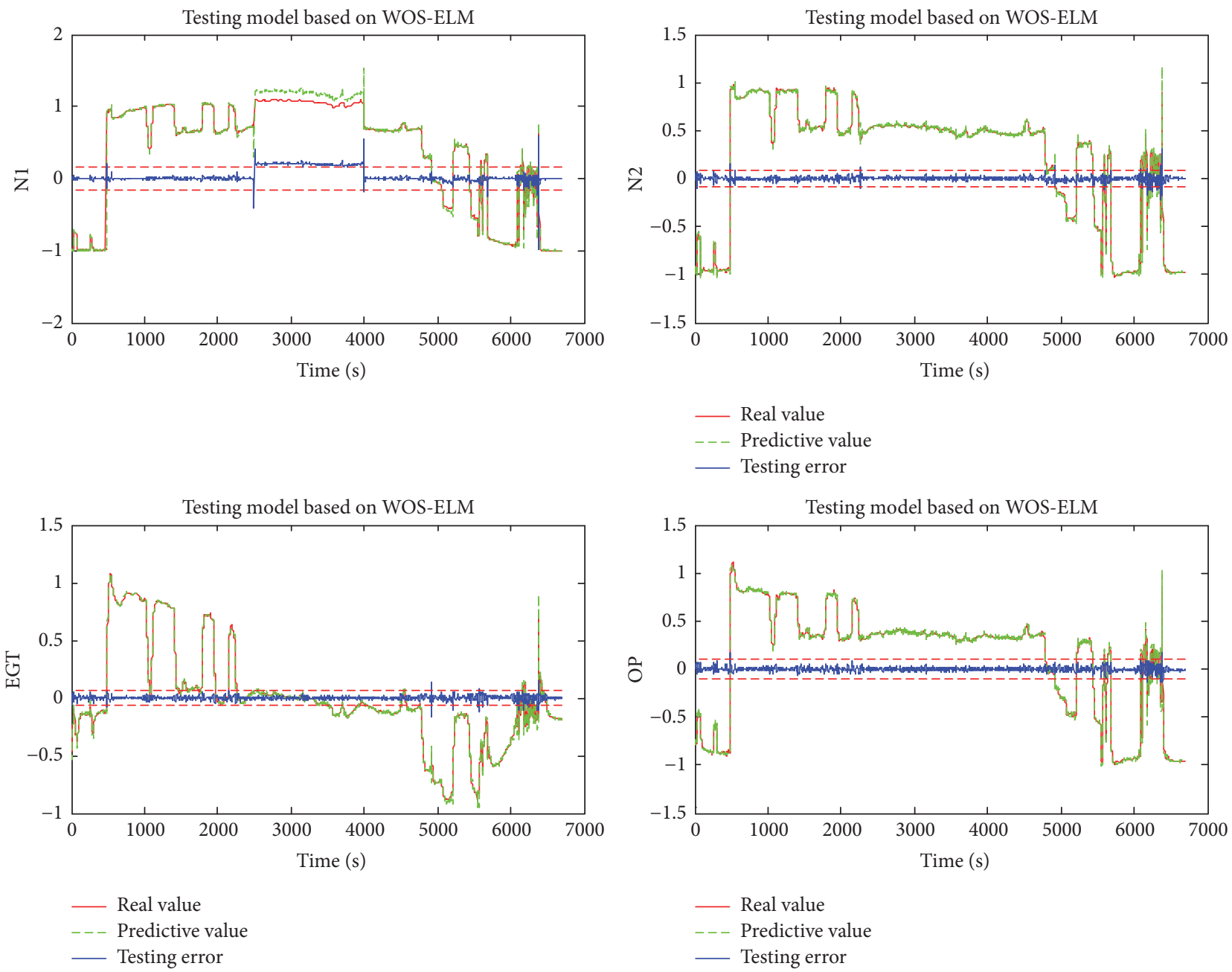

FIGURE 7: Results of time redundancy submodels for step fault of N1.

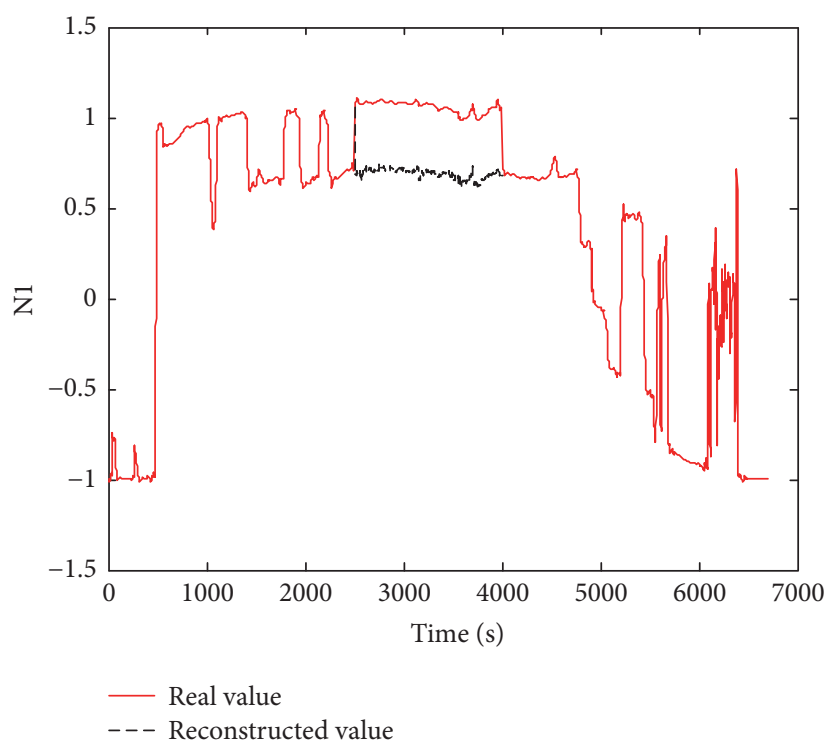

FIgURE 8: Reconstructed value for N1. where $Q_{k}=P_{k} H_{k+1}^{T}$. Finally, $\beta^{(k+1)}$ can be written as

$$
\beta^{(k+1)}=\beta^{(k)}+P_{k+1} H_{k+1}^{T}\left(Y_{k+1}-H_{k+1} \beta^{(k)}\right) .
$$

Step 3. Set $k=k+1$, go to Step 1 in the sequential learning phase, and continue sequential learning until finishing data learning.

\section{WOS-ELM-Based Double Redundancy FDR Method}

In this section, the proposed WOS-ELM is combined with double redundancy strategy to realize multiple sensors' FDR. Double redundancy refers to spatial redundancy and time redundancy. The former utilizes the redundancy information among all the sensors, while the latter uses the redundancy information of each sensor over consecutive time. In this paper, we set up two submodels for each sensor and use them to realize sensor fault diagnosis. The whole fault diagnosis process of the $n$th sensor is shown in Figure 1. From Figure 1, 

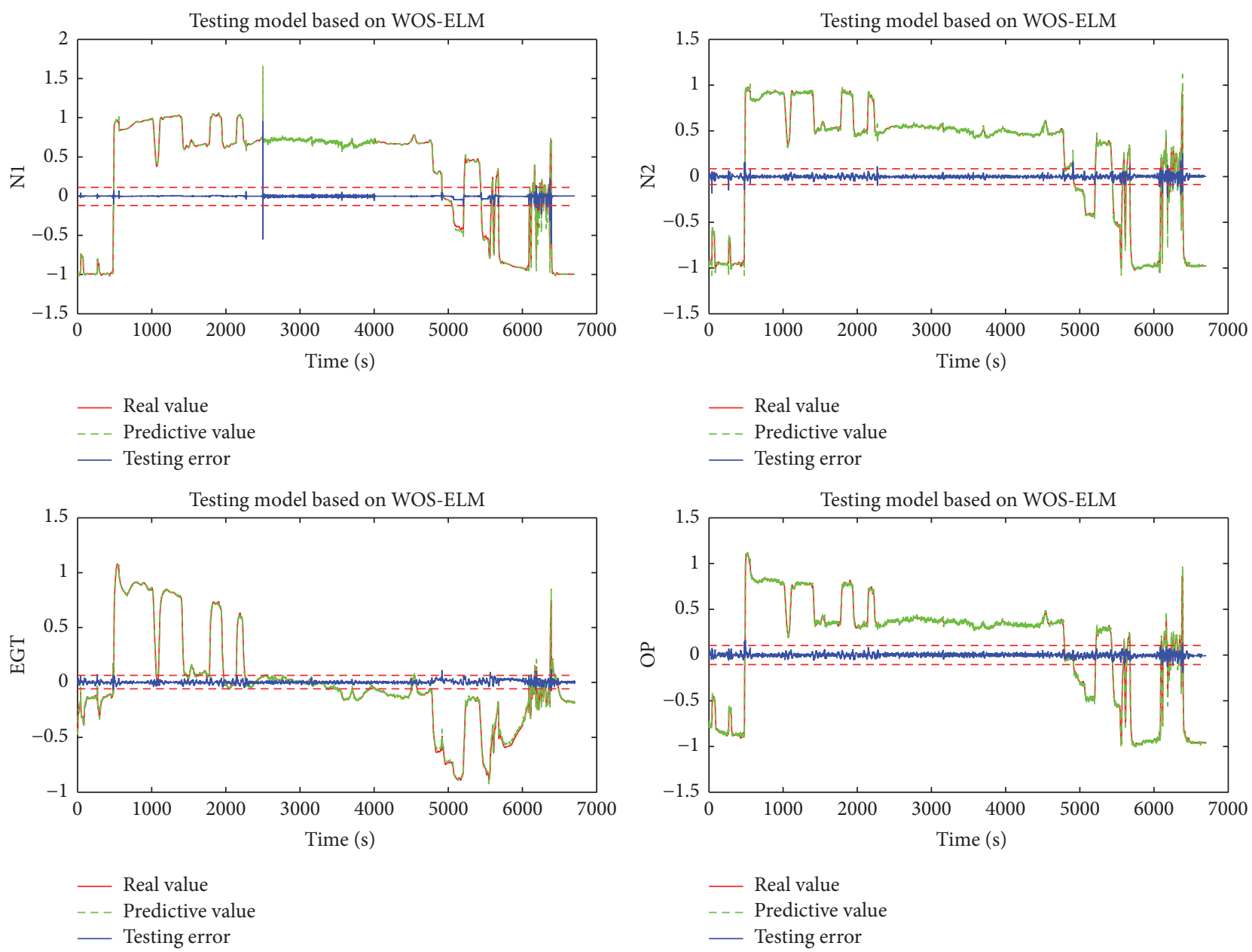

FIGURE 9: Time redundancy results after reconstruction.

it can be seen that the whole diagnosis process contains two stages, namely, offline modeling stage and online diagnosis stage.

3.1. Offline Modeling. The offline modeling stage of the proposed WOS-ELM-based double redundancy FDR is illustrated above the dashed line in Figure 1. The detailed description of the offline modeling stage is as follows.

(1) Before training the WOS-ELM model, wavelet denoising method is utilized to deal with the training data sampled from sensors. In this way, the training data is obtained.

(2) Maximum and minimum normalization method is used to normalize the denoised training data, and the data is standardized to be in $[-1,1]$.

(3) Input the normalized training data into spatial redundancy submodel and time redundancy submodel. For each spatial redundancy submodel, the input is the output of all sensors except the $n$th sensor at time $t$, and the output is the $n$th sensor's output at time $t$. For each time redundancy submodel, the input is the output of the $n$th sensor from time $t-p$ to $t-1$, and the output is the $n$th sensor's output at time $t$. Then, we can get $n$ well-trained spatial redundancy submodels and $n$ time redundancy submodels.
3.2. Online Diagnosis. When implementing the online monitoring, follow the following steps to realize the online detection of sensors' fault after receiving the present sensor data $x_{\text {new }}(1 \times n)$ in real time.

(1) The data sampled from the aeroengine sensors is firstly preprocessed as that at the offline modeling stage.

(2) Then, the preprocessed data is input into the welltrained spatial redundancy submodels and time redundancy submodels. These submodels' outputs are the evaluated value of each sensor at current time, from which we can calculate the residuals by comparing with its real value.

(3) Thresholds are used to determine whether there are sensors' faults. Here, thresholds, which have great influence on the accuracy of fault detection and diagnosis, should be set according to practical applications. In this paper, the residual sequence's standard variance while the system is under the normal situation is used to determine the thresholds. Generally, choose $m$ times of the standard variance. Assuming that the meaning of the residuals sequence is $\mu$, the standard variance is $\sigma$; then the range of the threshold can be set as $[\mu-m \sigma, \mu+m \sigma][14]$.

(4) As shown in Figure 1, when the spatial redundancy residuals and the time redundancy residuals both exceed their 

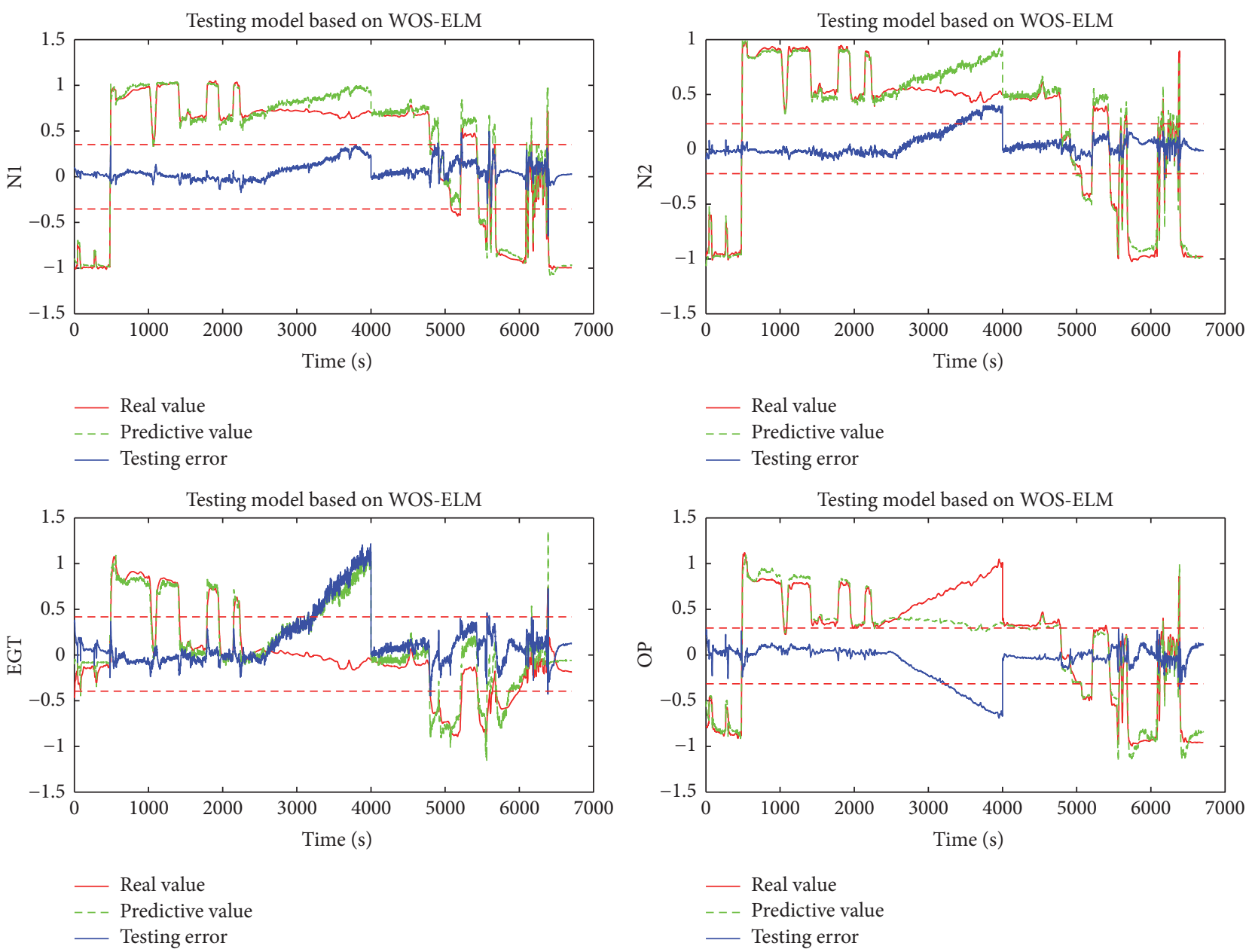

FIGURE 10: Spatial redundancy results for soft fault.

thresholds, a hard fault of the $n$th sensor occurs. Suppose the $n$th sensor encounters a hard fault. On the one hand, as the input of each spatial redundancy submodel is the other sensors' signals, which are all normal, the $n$th sensor's evaluated value is correct, while the other spatial redundancy submodels' outputs are not. Since the $n$th sensor's real value shows fault signals, and the other sensors do not, all the residuals exceed the thresholds. On the other hand, the time redundancy submodels could give us a definite answer of which sensor is running with fault. The sensor, whose time redundancy residual exceeds the threshold, is the faulty one. Furthermore, if there are more than one sensor falling in hard faults, all the spatial redundancy submodels' outputs exceed the corresponding thresholds. For time redundancy submodels, only the faulty sensor-related time redundancy residuals exceed the corresponding thresholds.

(5) If neither the spatial redundancy residuals nor the time redundancy residuals exceed the thresholds, but the change rate of the spatial redundancy residual exceeds the threshold for a long period of time, a soft fault may occur to the related sensor. Otherwise, it may be treated as a false alarm due to the change of flight phases. Considering that the change rate of residual is small for short period, the time interval is set to be $200 \mathrm{~s}$, and the expression is shown as

$$
c(i)=\varepsilon(i+200)-\varepsilon(i),
$$

where $\varepsilon(i)$ is the spatial redundancy residuals at time $i$.

If there are more than one sensor falling in soft fault, then the faulty sensor-related change rate of residual would exceed its corresponding thresholds.

(6) When the spatial redundancy residuals, the time redundancy residuals, and the change rate of spatial redundancy residual do not exceed the thresholds, it seems to be operated under normal status and there is no sensor fault.

During the FDR process, if the sensors' data are normal, the right data can be used as training data to make the spatial redundancy submodels and the time redundancy submodels more accurate and more suitable in the current flight phase. The trained process is depicted in Section 2.2.

After the faulty sensors have been detected and diagnosed, the faulty sensor-related time redundancy submodels' outputs can be used to reconstruct the fault signal. That is to say, we used trouble-free or reconstructed sensors' signals to update the training models, which make the diagnosis models 

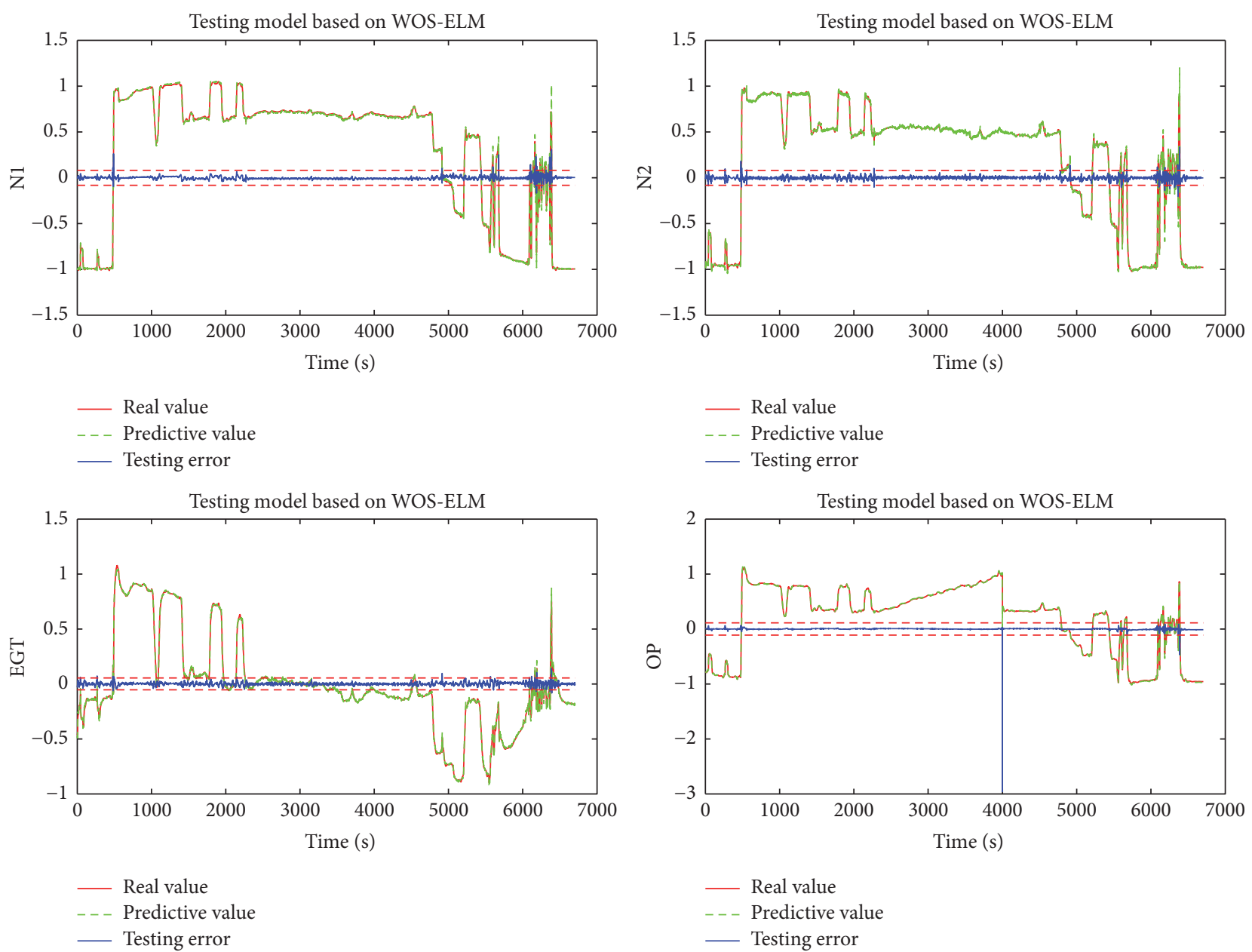

FIGURE 11: Time redundancy results for soft fault of OP.

much closer to the actual sensor operating environment. In order to ensure the diagnosis preciseness and system safety, the weight for reconstructed sensors' data should be appropriately reduced.

\section{Illustration and Discussion}

In this section, the data is acquired in real time from the sensors when the plane is in flight status. The simulation tool is MATLAB (R2010a). Four sensors' outputs are chosen in this paper, including low pressure rotor speed (N1), high pressure rotor speed (N2), engine exhaust temperature (EGT), and oil press (OP). The sampling time interval is 1 second. The number of the training data in the offline modeling is 6700 , and the number of the testing data in the online modeling is about 6700 .

4.1. Parameter Selection. In order to achieve better training effect, the activation function and the number of hidden layer neurons should be selected properly. To build the spatial redundancy submodels, this paper chooses $\mathrm{RBF}$, sin, and sig as activation function in WOS-ELM algorithm, and set $N_{0}=$ 800 and $N_{k+1}=6$ based on lots of test. The training accuracy
TABLE 1: Activation function and the number of hidden nodes for each sensor.

\begin{tabular}{lcc}
\hline Sensor & $\begin{array}{c}\text { Type of activation } \\
\text { function }\end{array}$ & $\begin{array}{c}\text { Number of hidden } \\
\text { layer neurons }\end{array}$ \\
\hline N1 & RBF & 70 \\
N2 & $\sin$ & 50 \\
EGT & $\sin$ & 60 \\
OP & $\sin$ & 30 \\
\hline
\end{tabular}

and testing accuracy with different activation functions can be seen in Figures 2 and 3.

In Figures 2 and 3 , the $x$-axis is the number of hidden nodes and the $y$-axis is the RMSE. From Figures 2 and 3, we can see that different activation functions have different influences on the accuracy of training process and testing process. In order to achieve higher modeling accuracy, the activation function and the number of hidden nodes for each sensor are selected according to a large number of trials, whose final results are shown in Table 1.

The training and testing results of these four sensors under normal state are shown in Figures 4 and 5. 

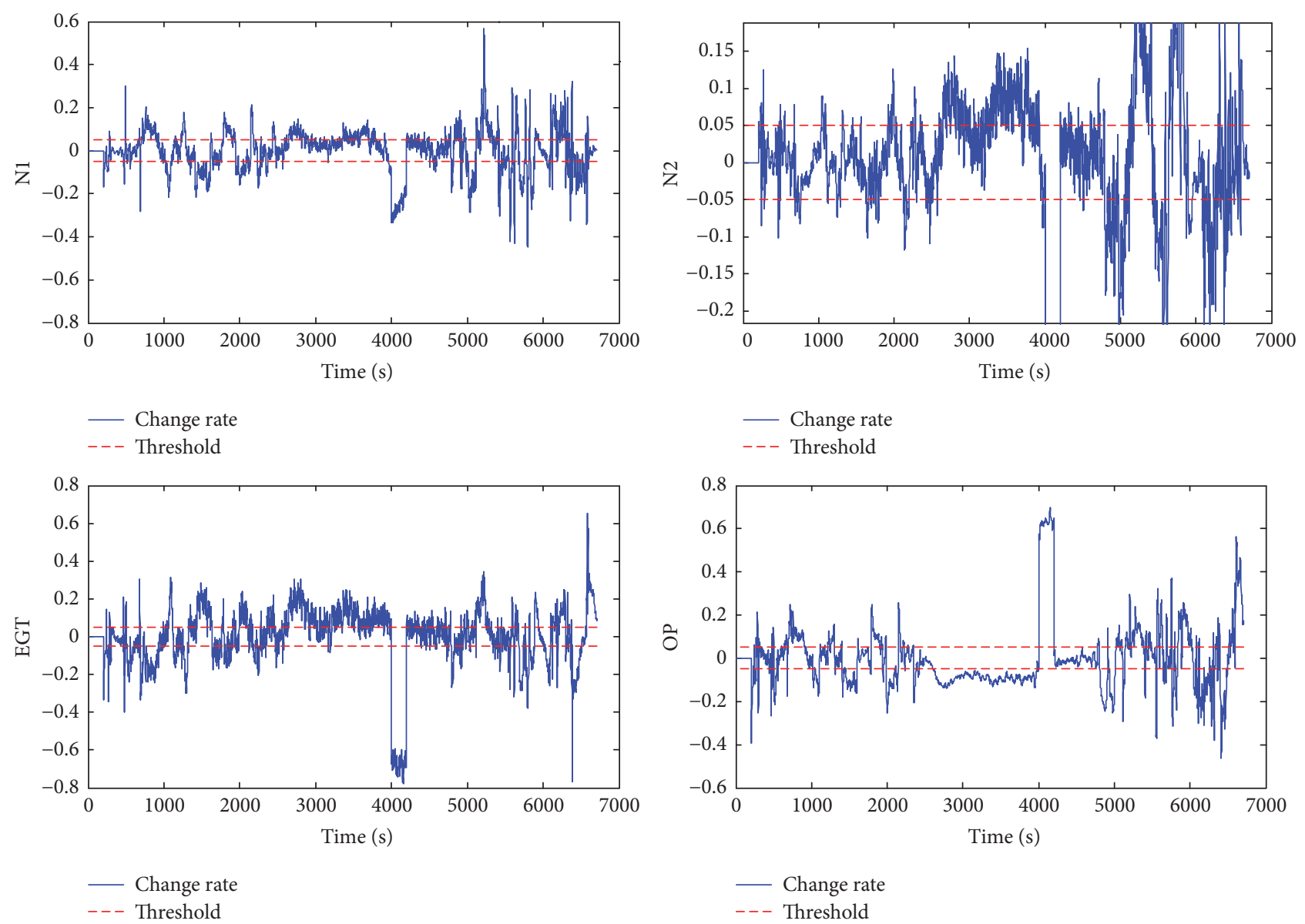

FIGURE 12: Change rate of spatial redundancy residuals.

Figure 4 shows that WOS-ELM algorithm has a good ability of linear fitting, and it can track sensors' output signal perfectly. That is to say, using this algorithm can get welltrained submodels.

Figure 5 shows that good effect can be received if these well-trained models are applied to evaluate the testing sensors' outputs, which is well used in the sensors' fault diagnosis process.

4.2. Hard Fault Diagnosis. Hard fault refers to instantaneous bigger step or mutations of the normal sensors' outputs, including step fault or pulse fault of sensors. Due to the fact that the real aeroengine sensors' faulty data is difficult to obtain for many reasons, for example, protection measures would be implemented when fault occurs, this paper adds a constant gain $\alpha$ to the original normal N1 signal to simulate step fault. In this paper, $\alpha$ is set to be 1.15 from $2500 \mathrm{~s}$ to $4000 \mathrm{~s}$. The results of the spatial redundancy submodels are shown in Figure 6.

Figure 6 shows that when the step fault occurs to N1, residuals of the four sensors all exceed their corresponding thresholds from $2500 \mathrm{~s}$ to time $4000 \mathrm{~s}$. Therefore, it cannot distinguish faulty sensor from the spatial redundancy results. As described in Section 3.2, the time redundancy models are introduced to determine which sensor is faulty. The results of the time redundancy submodels are shown in Figure 7.

From Figure 7, it is easy to see that the residual sequence of $\mathrm{N} 1$ sensor exceeds its threshold at $2500 \mathrm{~s}$, while the residuals of other sensors are within their corresponding thresholds. Combining spatial redundancy model and time redundancy model, we can conclude that N1 sensor encounters a hard fault at the $2500 \mathrm{~s}$. When sensors' faults are diagnosed, reconstruction should be done. The reconstruction of N1 can be seen in Figure 8.

Figures 8 and 9 show that WOS-ELM algorithm can be used to reconstruct fault signal, and the reconstructed signal can also be input into the well-trained models for further training.

4.3. Soft Fault Diagnosis. Soft fault refers to slow changing of the sensors' outputs, such as drift fault. In general, soft fault is mainly caused by aging and zero drift of the aeroengine sensor. In this paper, we can add a constant gain $\alpha$ and a constant deviation $\gamma$ for OP from 2500 s to 4000 s. The results of spatial redundancy submodels are shown in Figure $10(\alpha=$ $0.03, \gamma=0.05)$.

Compared to hard fault, it is not easy to diagnose soft fault due to tiny failure symptom at the early stage. In Figure 10, 

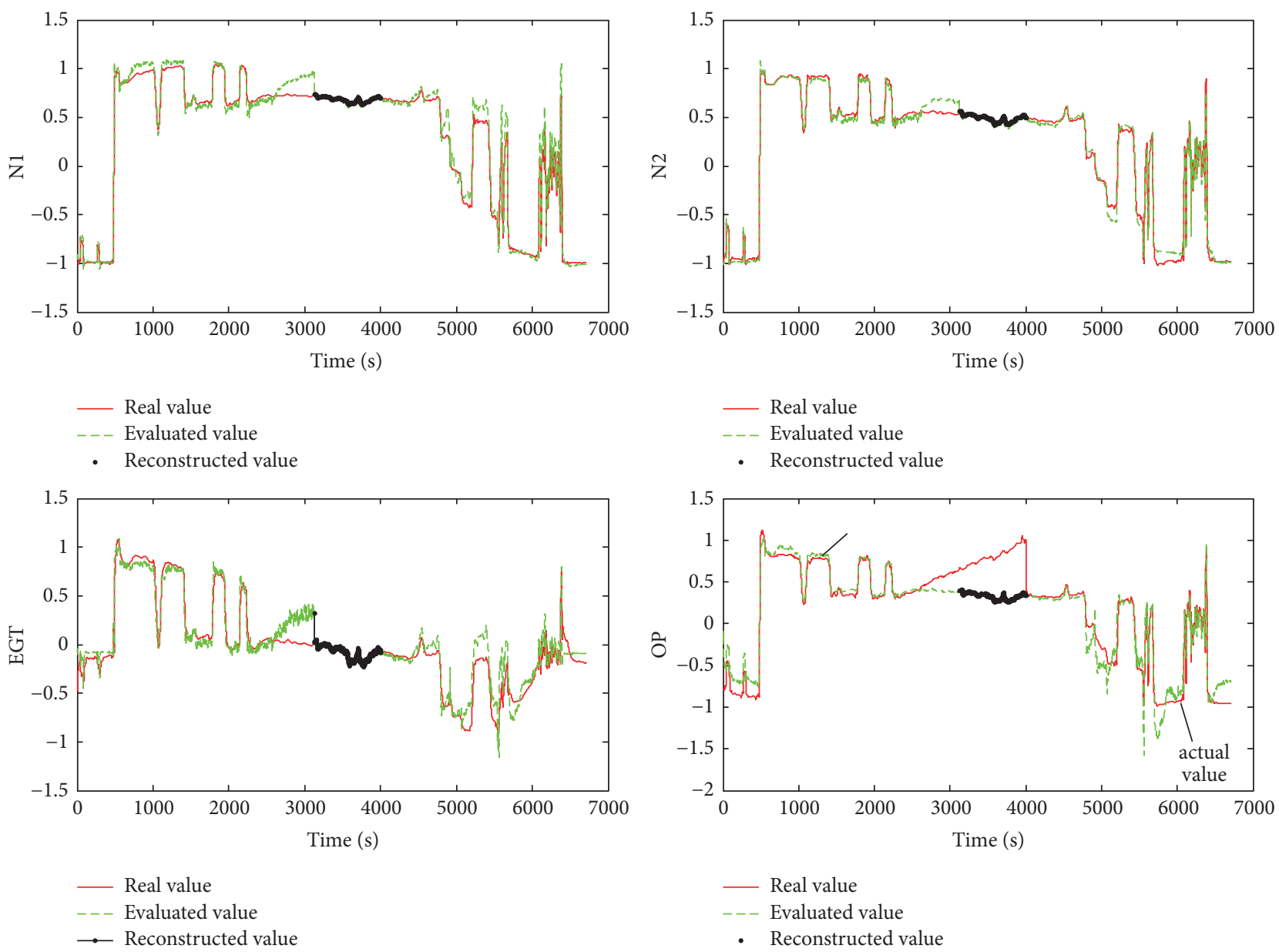

FIGURE 13: Reconstruction results.

it can be seen that all the spatial residuals are within the thresholds ranges until around $3300 \mathrm{~s}$. After around $3300 \mathrm{~s}$, all residuals exceed their corresponding thresholds except $\mathrm{N} 1$ (whose residual keeps growth and is near the threshold), which implies that some faults may occur. However, it is difficult to make decision of which sensor is faulty.

Turn to look at the time redundancy submodels' results, which are shown in Figure 11. From Figure 11, it is also difficult to determine the faulty sensor. As described in Section 3.2, we observe the change rate of the spatial redundancy residual, which is shown in Figure 12. In Figure 12, the thresholds are set to be -0.05 and 0.05 . Considering that the development of soft fault would go through a long period, there a soft fault occurs only when the duration of change rate exceeds the upper threshold or the lower threshold lasts for a long period. In this paper, the duration exceeding the threshold is set as 300 . Then, we can get the period of every sensor exceeding the thresholds for Figure 12, shown in Table 2.

In Table 2, “-” stands for never exceeding the threshold. According to Figure 12 and Table 2, we can confirm that the OP sensor has a soft fault at time $3125 \mathrm{~s}(2625+200+300)$. Here, 200 stands for the time lag for detecting the residual errors, and 300 stands for the duration time for residual
TABLE 2: The period of every sensor exceeding the thresholds.

\begin{tabular}{lcc}
\hline Sensor & $\begin{array}{c}\text { Exceed the upper } \\
\text { threshold period }\end{array}$ & $\begin{array}{c}\text { Exceed the lower threshold } \\
\text { period }\end{array}$ \\
\hline N1 & - & - \\
N2 & - & - \\
EGT & - & - \\
OP & - & $2625 \mathrm{~s} \sim 3923 \mathrm{~s}$ \\
\hline
\end{tabular}

change amplitude exceeding the thresholds. When detecting the soft fault, it may have continued for a period. However, considering that it had little effect on the flight of aircraft at the beginning of the soft fault, time delay for detecting soft fault is accepted.

Figure 13 illustrates the reconstructed results of the four sensors when detecting the soft fault of OP. After reconstruction, the results of its time redundancy submodel can be shown as in Figure 14. The change of the spatial redundancy residual is more efficiently applied to sensors' soft fault detection. 

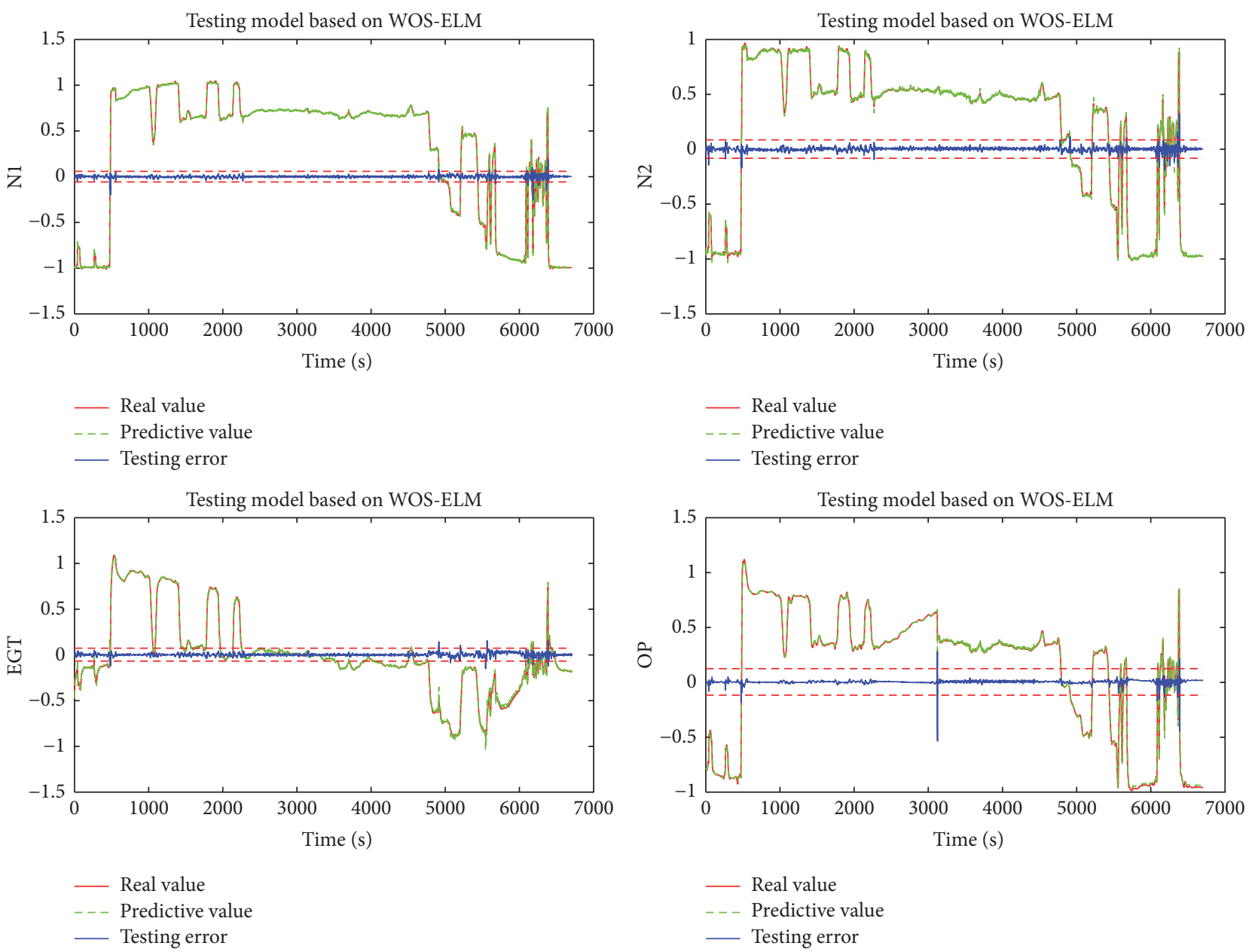

Figure 14: Time redundancy results after reconstruction.

\section{Conclusion}

In this paper, a double redundancy diagnosis approach based on WOS-ELM is proposed to diagnose sensor fault for the aeroengine. The proposed method contains two stages, that is, offline modeling stage and online detection stage. At the offline modeling stage, two series of models, namely, spatial redundancy submodels and time redundancy submodels, are trained to evaluate the sensors' value for further online detection, diagnosis, and reconstruction. At the online diagnosis stage, new sampled data is preprocessed to be input into these well-trained submodels. According to the outputs of these submodels and the practical sensors' value, several residuals are generated to diagnose sensors' faults. After that, WOSELM, which assigns different weights to the old and new data, is proposed to adjust parameters of ELM to improve the modeling accuracy. Simulation results show that the proposed method can be well used in sensors fault diagnosis.

The presented WOS-ELM-based double redundancy fault diagnosis and reconstruction method can also be extended using practical sampled sensors' outputs. Furthermore, the proposed method can be extended to other similar application fields.

\section{Conflicts of Interest}

The authors declare that there are no conflicts of interest regarding the publication of this paper.

\section{Acknowledgments}

This work was supported by the National Science Foundation for Young Scientists of China (Grant no. 61703406), the project 973 (Grant no. 2014CB744904), civil aviation science and technology major projects (Project no. MHRD20130112), Joint Funds of the National Natural Science Foundation of China and Civil Aviation Administration of China Key Project (Grant no. U1533201), and the Fundamental Research Funds for the Central Universities (Project no. 3122014C010, 3122015C011).

\section{References}

[1] K. Cai L, Y. Sun F, and W. Yao W, "Fault diagnosis and adaptive reconfiguration control for sensors in aeroengine," DIanguang YU Kongzhi, vol. 16, no. 6, pp. 57-61, 2009. 
[2] B. Zhao W, "Research on aircraft engine sensor fault diagnosis and signal reconstruction," Nanjing University of Aeronautics and Astronautics, pp. 34-42, 2011.

[3] K. Takahisa and S. Donald L, "Application of a bank of Kalman filters for aircraft engine fault diagnosis," Atlanta: ASME Turbo Expo Power for Land, Sea, and Air, pp. 1-10, 2003.

[4] X. Lishuang, C. Tao, and D. Fang, "Sensor fault diagnosis based on least squares support vector machine online prediction," in Proceedings of the 2011 IEEE 5th International Conference on Robotics, Automation and Mechatronics, (RAM '11), pp. 275-279, Qingdao, China, September 2011.

[5] S. Duan, Q. Li, and Y. Zhao, "Fault diagnosis for sensors of aeroengine based on improved least squares support vector regression," in Proceedings of the 2011 8th International Conference on Fuzzy Systems and Knowledge Discovery, FSKD 2011, Jointly with the 2011 7th International Conference on Natural Computation, (ICNC '11), pp. 1962-1966, Shanghai, China, July 2011.

[6] H. Zhang, T. Chen, and W. Li, "Abrupt sensor fault diagnosis based on wavelet network," in Proceedings of the 2006 IEEE International Conference on Information Acquisition, (ICIA '06), pp. 111-116, Weihai, China, August 2006.

[7] X. Wang M and Z. Ren, "a sensor fault diagnosis method research based on wavelet transform and hilbert-huang transform," Hong Kong: Conference on Measuring Technology and Mechatronics Automation, pp. 81-84, 2013.

[8] G. B. Huang, Q. Y. Zhu, and C. K. Siew, "Extreme learning machine: a new learning scheme of feedforward neural networks," in Proceedings of the IEEE International Joint Conference on Neural Networks, vol. 2, pp. 985-990, Budapest, Hungary, July 2004.

[9] Y. Sun G, J. Liu Y, and Z. Zhan, "Extreme learning machinebased aircraft engine sensor fault diagnosis," Sensor and Micro System, vol. 33, pp. 23-26, 2014.

[10] X. Zhang and L. Wang H, "Have a choice and forgetting mechanism of extreme learning machine in the application of time series prediction," Acta Physica Sinica, vol. 60, no. 8, Article ID 080504, 2011.

[11] N. Liang, G. Huang, P. Saratchandran, and N. Sundararajan, "A fast and accurate online sequential learning algorithm for feedforward networks," IEEE Transactions on Neural Networks and Learning Systems, vol. 17, no. 6, pp. 1411-1423, 2006.

[12] G. Yin, Y. Zhang T, and N. Li Z, "Fault diagnosis method based on online sequential extreme learning machine," Vibration, Measurement and Diagnosis, vol. 33, no. 2, pp. 325-329, 2013.

[13] B. Yu J and L. Zhu Y, "Transformer fault diagnosis using weighted extreme learning machine," Computer Engineering and Design, vol. 34, pp. 4340-4344, 2013.

[14] B. Y. Li, Q. H. Li, J. K. Wang, and X. H. Huang, "Sensor fault adaptive diagnosis of aero-engines based on ImOS-ELM," in Hangkong Xuebao, vol. 34, pp. 2316-2324, 10 edition, 2013.

[15] B. Mirza, Z. Lin, and K.-A. Toh, "Weighted online sequential extreme learning machine for class imbalance learning," Neural Processing Letters, vol. 38, no. 3, pp. 465-486, 2013.

[16] B. Mirza, Z. Lin, J. Cao, and X. Lai, "Voting based weighted online sequential extreme learning machine for imbalance multi-class classification," in Proceedings of the IEEE International Symposium on Circuits and Systems, (ISCAS '15), pp. 565568, Lisbon, Portugal, May 2015. 


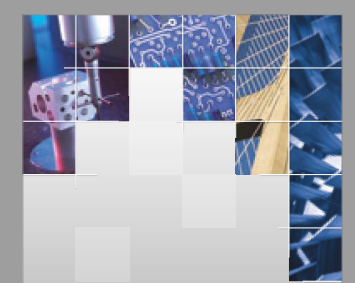

\section{Enfincering}
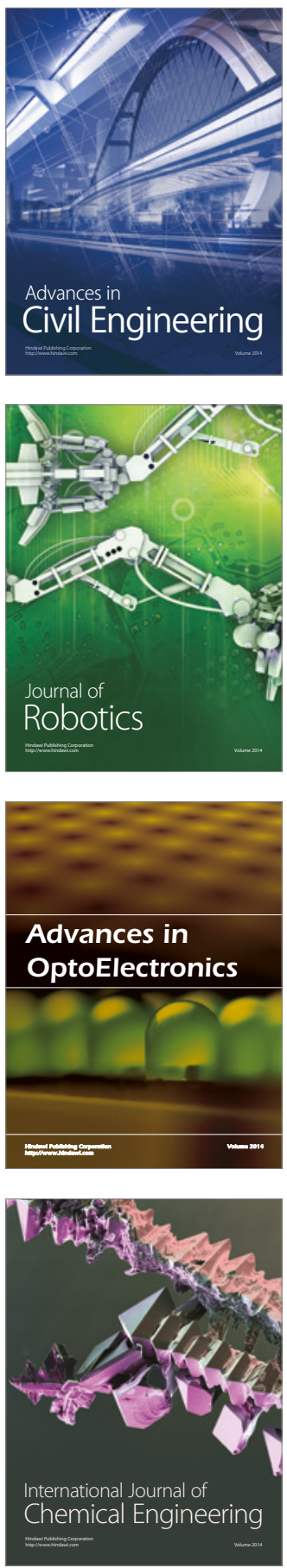

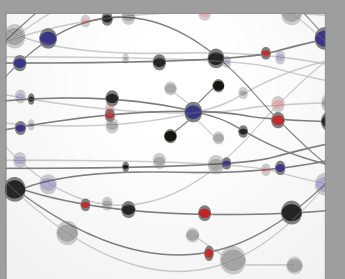

The Scientific World Journal

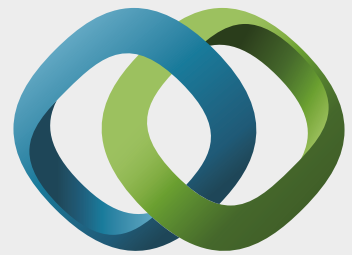

\section{Hindawi}

Submit your manuscripts at

https://www.hindawi.com
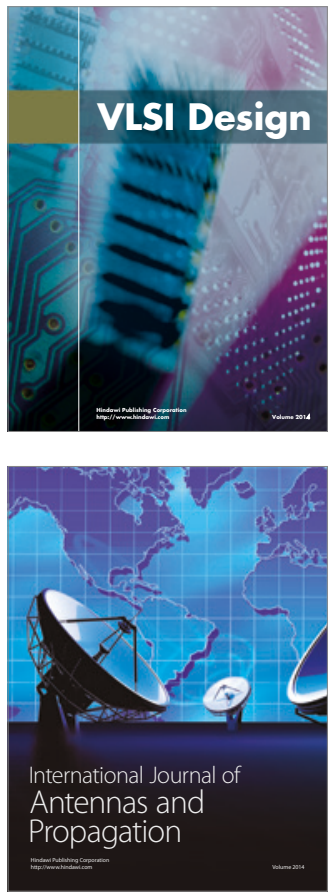

\section{Rotating}

Machinery
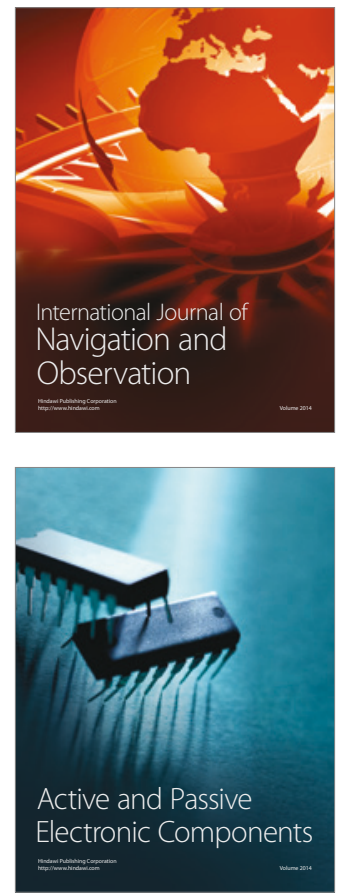
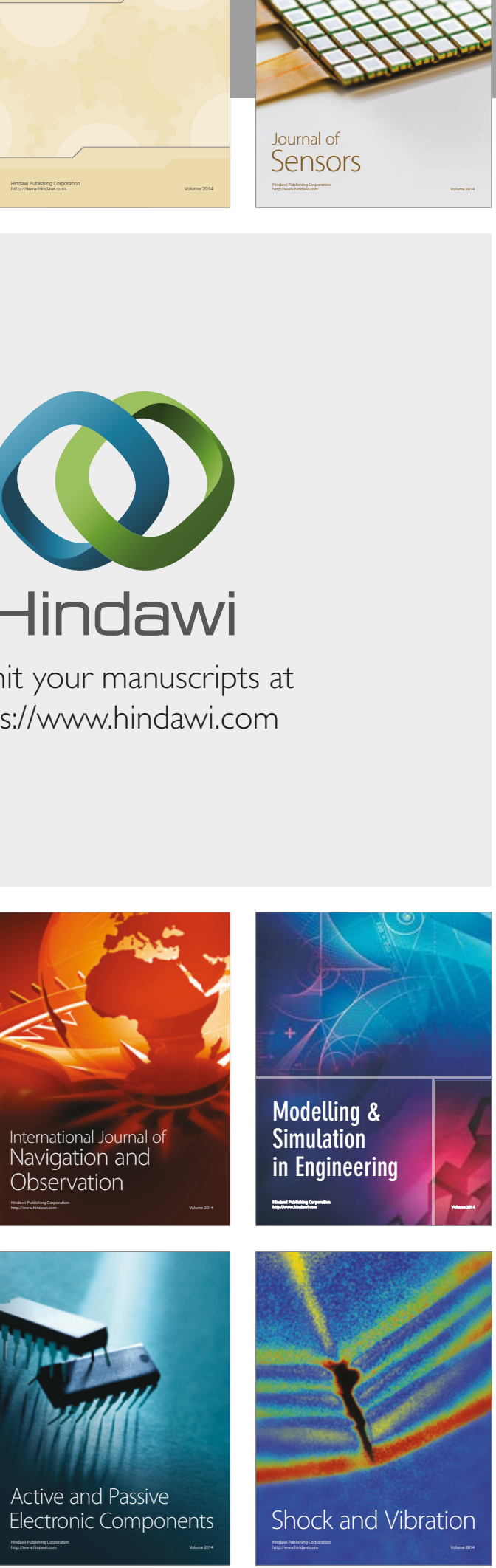
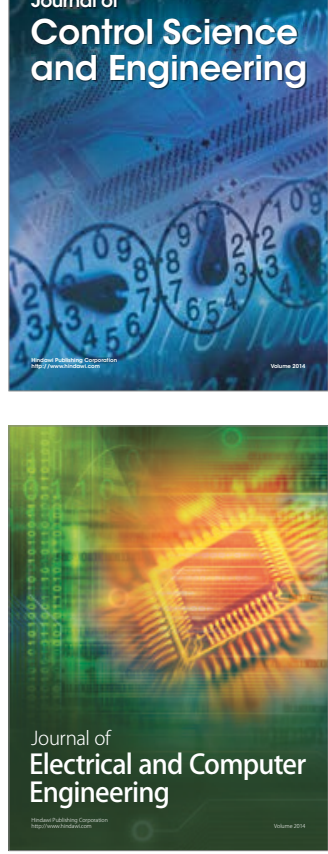

Distributed

Journal of

Control Science

and Engineering
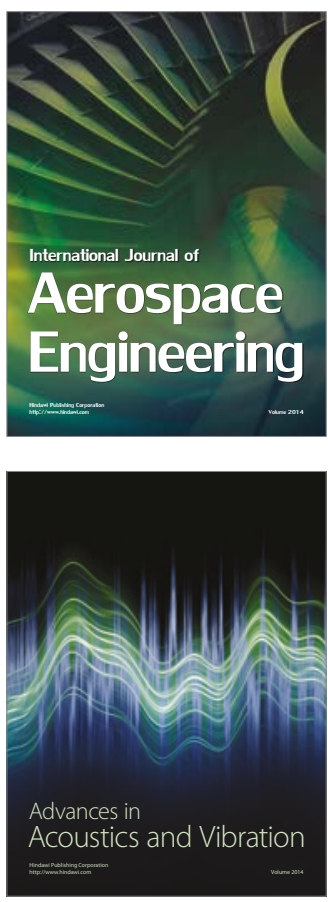

Sensor Networks 